\title{
Altura dominante e índice de sítio para Pinus taeda em dois polos de desenvolvimento florestal no sul do Brasil
}

\author{
Dominant height and site index for loblolly pine (Pinus taeda L.) in two \\ forestry clusters in the Southern Region of Brazil
}

\author{
Mariana Smanhotto Schuchovski ${ }^{*}$, Julio Eduardo Arce ${ }^{2}$ \\ e Edilson Batista de Oliveira ${ }^{3}$
}

\begin{abstract}
RESUMO
Para o planejamento florestal em médio e longo prazo, é necessário conhecer a estrutura e a dinâmica dos povoamentos florestais que serão responsáveis por fornecer matéria-prima às indústrias de base florestal. A classificação dos sítios para o conhecimento da qualidade e produtividade potencial das áreas florestais são fatores cruciais para a tomada de decisões no manejo florestal, orientando sobre os locais onde serão realizados investimentos onde povoamentos serão implantados. O objetivo deste estudo foi ajustar, comparar e avaliar o comportamento de três modelos de índice de sítio para plantações de Pinus taeda localizadas em dois polos de desenvolvimento florestal nos estados do Paraná e de Santa Catarina, na Região Sul do Brasil, utilizando funções de altura dominante e sítio. Para descrever a relação entre as variáveis altura dominante e idade, e classificar as áreas quanto ao seu potencial de produtividade foram ajustadas as seguintes funções de sítio: de Chapman-Richards, de Prodan e de Schumacher. O modelo de Chapman-Richards apresentou superioridade em relação aos demais, sendo que as curvas resultantes apresentaram padrão anamórfico e estabilidade. Em comparação com curvas ajustadas por outros autores para a mesma espécie e para regiões próximas, a curva ajustada neste estudo estima maiores alturas dominantes do que alguns estudos mais antigos e, alturas dominantes menores ou iguais a alguns estudos mais atuais.
\end{abstract}

Paravras-chave: Crescimento; Modelos alométricos; Produtividade.

\begin{abstract}
For forest planning in the medium and long term, it is necessary to know the structure and dynamics of forest stands that will be responsible for supplying raw materials to forest-based industries. The classification of the sites for the knowledge of the quality and the potential productivity of the forest areas are crucial for the decision-making in forest management, orienting on the places to make the investments to stablish forest stands. The goal of this study was to adjust, compare and evaluate the behavior of three site index models for Pinus taeda plantations located in two forestry development zones in Paraná and Santa Catarina states, Southern Region of Brazil, using dominant height and site functions. To describe the relationship between the dominant height and age variables and then to classify the areas as to their potential productivity, the following site functions were adjusted: Chapman-Richards, Prodan and Schumacher. The Chapman-Richards model was superior to the others, and the resulting curves presented anamorphic pattern and stability. The curve fitted in this study estimates higher dominant heights than some previous studies, and dominant heights less than or equal to some more current studies.
\end{abstract}

Keywords: Growth; Yield; Loblolly pine.

\section{INTRODUÇÃO}

Para uma tomada de decisões criteriosa em manejo florestal é necessário compreender os padrões de crescimento de uma espécie e avaliar a qualidade de sítio (AVERY; BURKHART, 1994), definida como a soma dos fatores edáficos, biológicos e climáticos que afetam as plantas, dentre os quais um ou mais são dominantes (SPURR, 1952). No contexto do manejo para produção de madeira,

1. Verde Floresta Consultoria Ambiental e Florestal. Curitiba / PR, Brasil. * Autor Correspondente: marischu@hotmail.com

2. Departamento de Ciências Florestais, Universidade Federal do Paraná - UFPR. Curitiba / PR, Brasil.

3. Unidade de Florestas, Empresa Brasileira de Pesquisa Agropecuária - Embrapa. Colombo / PR, Brasil.

Sci. For., Piracicaba, v. 47, n. 121, p. 92-104, mar. 2019 DOI: dx.doi.org/10.18671/scifor.v47n121.09 
Clutter et al. (1983) definem a qualidade do sítio como o potencial de produção de madeira de um local, para árvores de uma determinada espécie ou tipo de floresta. Assim, a qualidade do sítio tem significado apenas no que diz respeito a uma ou mais espécies que podem ser consideradas em um local particular.

A qualidade do sítio é frequentemente estimada pelo crescimento em altura, que é uma variável altamente correlacionada com a produtividade volumétrica e pouco influenciada pela variação da densidade do povoamento e tratos silviculturais (desbastes seletivos por baixo). O indicador mais comum da qualidade de sito é o índice de sítio, definido como a altura média das árvores dominantes de parte de um povoamento (altura dominante) a uma determinada idade, chamada idade índice. Diversas definições para altura dominante são aplicadas no mundo e embora não haja consenso sobre a definição de quais e quantas árvores serão incluídas, todas consideram a medição de árvores da parte superior do dossel, ou seja, das classes dominantes e codominantes (BURKHART; TOMÉ, 2012; CLUTTER et al., 1983; SANQUETTA et al., 2014).

Segundo Assmann (1970), o crescimento em altura das árvores segue um curso regular em conformidade com certas leis naturais. Na curva de incremento em altura ocorrem dois pontos de inflexão e um ponto de culminação que ocorre na mesma idade que o ponto de inflexão na curva de crescimento.

Em pleno sol, o crescimento em altura é o mesmo para uma mesma espécie sob condições similares de sítio, podendo variar anamórfica ou polimorficamente (OLIVER; LARSON, 1996). Gerar curvas de índice de sítio anamórficas é considerar que as diferenças em sítios resultam em diferenças similares nas taxas de crescimento em altura para todas as idades, partindo do princípio que a altura é uma função linear da idade. Por outro lado, as curvas de índice de sítio polimórficas, desenvolvidas por Osborne e Schumacher (1935) partem do pressuposto que o crescimento das árvores varia de sítio para sítio (CLUTTER et al., 1983; MACHADO, 1980).

As curvas de índice de sítio podem ser construídas tanto para mostrar o desenvolvimento de um povoamento que atinge uma determinada altura a uma determinada idade, como para a finalidade de classificação. A forma das curvas resultantes depende, até certo ponto, do tipo de função utilizada para o ajuste. Assim, diante da grande amplitude de variação biológica que ocorre naturalmente, é importante que a função seja bastante flexível (STRAND, 1964).

Clutter et al. (1983) comentam que a base para a construção das curvas de índice de sítio é que a altura dominante média das árvores da parcela permaneça numa mesma classe de sítio durante toda a sua vida, para que esta classificação propicie um suporte confiável para os estudos de crescimento e produção florestal. Entretanto, Avery e Burkhart (1994) afirmaram que o índice de sítio não é uma constante, mudando periodicamente em consequência de variações ambientais e climáticas. O seu valor para uma determinada espécie não pode ser utilizado para diferentes espécies no mesmo sítio.

Apesar de algumas limitações, o índice de sítio é o método mais utilizado para definir a potencialidade dos sítios florestais e assim o será até que métodos que conjuguem a soma de vários fatores ambientais possam ser traduzidos de forma numérica mais correta e acessível aos usuários do setor (CUNHA NETO, 1994).

No Brasil, as plantações florestais de Pinus spp. ocupam 1,6 milhão de hectares e concentram-se nos estados do Paraná (42\%) e em Santa Catarina (34\%) (IBÁ, 2016), sendo que nestes estados a espécie representa a maior parte da área de plantações FLORESTAIS. Nas últimas décadas, foram desenvolvidos diversos estudos para plantações de Pinus taeda na Região Sul do Brasil, desde a classificação de sítios (BOGNOLA, 2007; COSTA; ARCE; ANGELO, 2014; MACHADO, 1980; OLIVEIRA; AHRENS, 1987; OLIVEIRA; OLIVEIRA; HAFLEY, 1989; SCHUCHOVSKI, 2014; SCOLFORO; MACHADO, 1988a), até o desenvolvimento de modelos de simulação e prognose do crescimento para diversas finalidades tais como (AHRENS, 1992; ARCE, 2000; ARCE et al., 1998; 1997; OLIVEIRA, 1995; SCOLFORO; MACHADO, 1996; SCOLFORO, MAESTRI; MACHADO, 1997; RETSLAFF, 2014; SCHUCHOVSKI, 2003; TEMPS, 2005).

O objetivo deste estudo foi ajustar, comparar e avaliar o comportamento de modelos de índice de sítio para plantações de Pinus taeda localizadas em dois polos de desenvolvimento florestal nos estados do Paraná e de Santa Catarina, na Região Sul do Brasil, utilizando funções de altura dominante e sítio para descrever a relação entre as variáveis altura dominante e idade e classificar as áreas quanto ao seu potencial de produtividade. 
Schuchovski et al. - Altura dominante e índice de sítio para Pinus taeda em dois polos de desenvolvimento florestal no sul do Brasil

\section{MATERIAL E MÉTODOS}

\section{Caracterização Ecológica e Silvicultural da Área}

O estudo foi realizado em plantações de Pinus taeda pertencentes às empresas Florestal Vale do Corisco S.A. e Renova Floresta, à época administradas pela empresa Valor Florestal. Estes povoamentos abrangem dois polos de desenvolvimento florestal: o de Jaguariaíva, no Paraná e, o de Rio Negrinho, em Santa Catarina. Ao total, as plantações estão localizadas em 34 municípios: 22 no Sudeste e Nordeste do Paraná, cinco no Norte de Santa Catarina e sete no Sudoeste de São Paulo (FIGURA 1).

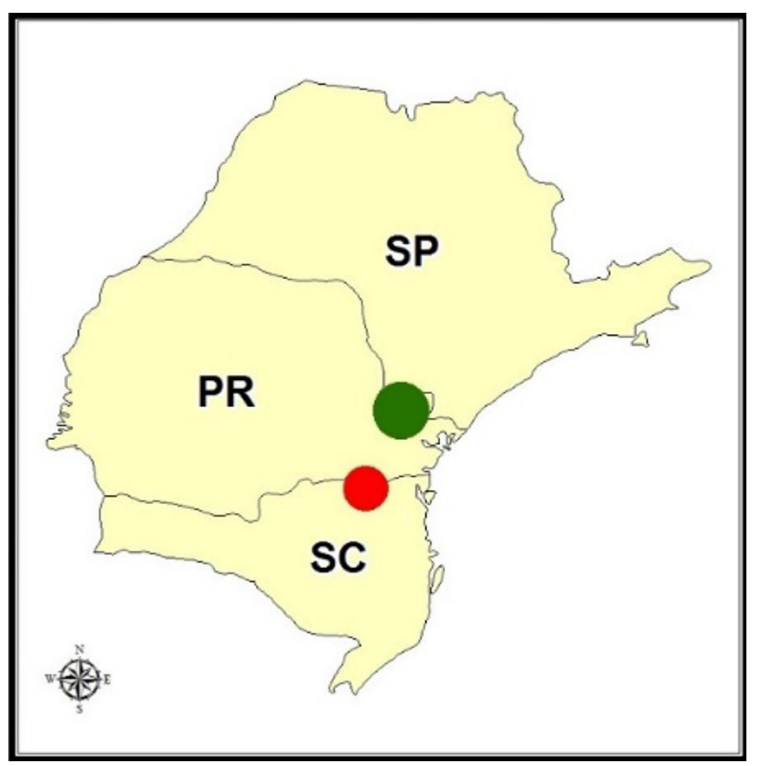

FIGURA 1 - LOCALIZAÇÃO DOS POVOAMENTOS DE Pinus taeda EM ESTUDO

FIGURA 1 - STUDIED Pinus taeda FOREST PLANTATIONS LOCATION

Os plantios se situam entre as latitudes $23^{\circ} 49^{\prime} 10^{\prime \prime} \mathrm{S}$ e $26^{\circ} 26^{\prime} 80^{\prime \prime} \mathrm{S}$ ao Sul e as longitudes $50^{\circ} 36^{\prime} 23^{\prime \prime} \mathrm{O}$ e $48^{\circ} 43^{\prime} 30^{\prime \prime} \mathrm{O}$ a Oeste, apresentando dois tipos de clima, Cfa e Cfb, de acordo com a classificação de Köppen (1936). A precipitação média anual varia entre 1200 e $1400 \mathrm{~mm}$, e parte nas faixas entre 1400 e 1600 mm e, 1600 e 1800 mm (IAPAR, 2000). Segundo a classificação da vegetação do Instituto Brasileiro de Geografia e Estatística - IBGE, a área está inserida nos biomas Mata Atlântica e Cerrado, abrangendo principalmente a vegetação secundária de Floresta Ombrófila Mista e Savana, sendo uma pequena parte de Floresta Ombrófila Densa (Floresta Tropical Pluvial) (BRASIL, 2004a;b). A região abrange grande diversidade de classes de solos, sendo os principais: cambissolos húmicos, cambissolos háplicos, neossolos regolíticos, argissolos vermelho-amarelos, latossolos brunos, latossolos vermelhos, latossolos vermelho-amarelos (EMBRAPA, 2008).

Foram utilizados dados de 1472 parcelas permanentes de inventário florestal contínuo, instaladas em povoamentos comerciais estabelecidos entre 1972 e 2005, com idades entre 3,7 e 33,2 anos, medidas no período entre 2000 e 2009, compreendendo 220.908 árvores. As variáveis medidas foram: CAP (Circunferência à Altura do Peito) com casca, em centímetros, de todas as árvores das parcelas; e altura total, em metros, de $20 \%$ das árvores vivas de cada parcela além de árvores dominantes e co-dominantes, sendo as árvores de maiores DAP da parcela, conforme Assmann (1970).

A densidade de plantio destes povoamentos é de 1666 árv./ha (espaçamento de 3,0 x 2,0 m), para áreas implantadas antes de 2003. Após 2003, a densidade de plantio passou a ser de 1333 árv./ha (espaçamento de $3,0 \times 2,5 \mathrm{~m}$ ) para os plantios realizados em SC. O DAP médio das árvores do povoamento é de $21,7 \mathrm{~cm}$, variando entre $1,3 \mathrm{~cm}$ e $72,3 \mathrm{~cm}$. A altura total média das árvores do povoamento é de 17,3 , variando entre $1,6 \mathrm{~m}$ e $38,3 \mathrm{~m}$.

\section{Modelos de altura dominante e índice de sítio}

Para a determinação do índice de sítio de cada parcela, utilizou-se a sua altura dominante definida pelo critério de Assman/Hummel (ASSMANN, 1970) como a altura média das 100 árvores de maior diâmetro por hectare. 
Para a estimativa da altura dominante e posterior classificação de sítio das parcelas da área em estudo foram ajustados três modelos: de Chapman-Richards (RICHARDS, 1959; CHAPMAN, 1961), de Prodan (1968), e de Schumacher (1939), apresentados na TABELA 1.

TABELA 1 - MODELOS TESTADOS PARA ESTIMAR ALTURA DOMINANTE E ÍNDICE DE SÍTIO

TABLE 1 - TESTED MODELS TO ESTIMATE DOMINANT HEIGHT AND SITE INDEX

\begin{tabular}{ccc}
\hline FUNÇÃO & FORMA UTILIZADA & FONTE \\
\hline Chapman-Richards & $h_{d o m}=b_{0}\left[1-e^{\left(-b_{I} I\right)}\right]^{b_{2}}+\varepsilon_{i}$ & Richards (1959), Chapman (1961) \\
Prodan & $h_{\text {dom }}=\frac{I^{2}}{b_{0}+b_{1} I+b_{2} I^{2}}+\varepsilon_{i}$ & Prodan (1968) \\
Schumacher & $h_{\text {dom }}=b_{0} * e^{\left[b_{l}\left(\frac{I}{I}\right)\right]}+\varepsilon_{i}$ & Schumacher (1939), Burkhart e Tomé (2012)
\end{tabular}

em que: $h_{\text {dom }}=$ altura dominante $(m) ; I=$ idade $($ anos $) ; b_{0}, b_{1}, b_{2}=$ coeficientes a serem estimados $; e=$ constante de Euler $; \varepsilon_{i}=$ erro

Para estes ajustes, foram utilizados 1493 pares de valores altura-idade, representadas pelas alturas dominantes de cada parcela, com idades entre 3,6 e 32,2 anos.

A escolha do modelo mais adequado foi baseada nas seguintes estatísticas de regressão: valor de $F$, coeficientes de determinação ajustado (R2aj), erro padrão da estimativa em percentagem (Syx\%), além da análise visual do gráfico de ajuste e da distribuição gráfica dos resíduos (\%).

Utilizou-se o método da curva guia para a construção das curvas de índice de sítio anamórficas (CLUTTER et al., 1983), seguindo como base a altura dominante à idade índice, ou idade de referência, de 15 anos. O número de classes de sítio, bem como o intervalo entre as classes, foi definido utilizando-se como parâmetro a amplitude dos valores de altura dominantes (limites inferior e superior). Selecionouse a equação ajustada que obteve o melhor desempenho na estimativa da altura dominante em função da idade para construir uma tabela de índice de sítio, bem como as curvas de índice de sítio. Com o modelo selecionado, estimou-se o índice de sítio das parcelas para a sua posterior classificação.

\section{RESULTADOS E DISCUSSÃO}

A FIGURA 2 apresenta o gráfico de dispersão da altura dominante (em metros) versus idade (em anos) para o conjunto de dados utilizado no ajuste, no qual se observa a típica curva em forma sigmoidal-côncava. Observa-se que o conjunto de dados em estudo abrange uma grande amplitude de dimensões de árvores, inclusive árvores com diâmetro e altura próximos aos valores assintóticos.

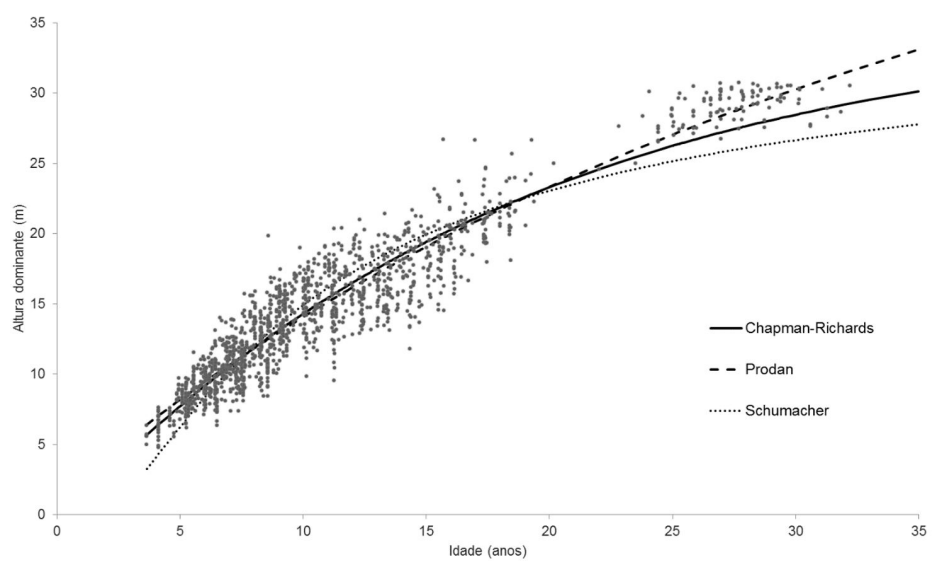

FIGURA 2 - CURVAS DE SÍTIO AJUSTADAS COM DIFERENTES MODELOS FIGURE 2 - FITTED SITE CURVES WITH DIFFERENT MODELS

Assim, embora algumas idades intermediárias não estejam representadas, o conjunto de dados altura-idade pode ser considerado bastante satisfatório para a realização do ajuste dos modelos para a área do estudo. Na mesma Figura, são apresentadas as curvas resultantes do ajuste das funções de 
Schuchovski et al. - Altura dominante e índice de sítio para Pinus taeda em dois polos de desenvolvimento florestal no sul do Brasil

sítio para os polos de desenvolvimento florestal de Jaguariaíva, Paraná, e de Rio Negrinho, Santa Catarina, na qual se observam comportamentos bastante distintos das curvas, seguindo tendências independentes, cruzando-se em alguns pontos.

A função de Chapman-Richards apresentou dificuldades de convergência, gerando valores assintóticos irreais sob o ponto de vista biológico, optando-se, assim, fixar o parâmetro $b 0$ em 35 . Para as funções de Schumacher e de Prodan não foi necessário este procedimento, uma vez que o ajuste destas funções aos dados não apresentou dificuldades.

A TABELA 2 resume os parâmetros estimados e os resultados das estatísticas de regressão obtidos com os modelos de sítio ajustados para os dados de altura-idade que representam os polos de desenvolvimento florestal de Jaguariaíva, no Paraná e de Rio Negrinho, em Santa Catarina. As estatísticas de regressão são apresentadas em ordem decrescente do coeficiente de determinação (R2aj) e crescente do erro padrão de estimativa (Syx\%).

TABELA 2 - PARÂMETROS ESTIMADOS E ESTATÍSTICAS DE REGRESSÃO $R_{a j}^{2} S_{y x} \%$ E F DOS MODELOS DE SÍTIO AJUSTADOS

TABLE 2 - ESTIMATED PARAMETERS AND REGRESSION ESTATISTICS $R^{2}{ }_{j j^{\prime}} S_{y x} \%$ AND F FOR THE ADJUSTED SITE MODELS

\begin{tabular}{|c|c|c|c|c|c|c|c|}
\hline \multirow{2}{*}{ FUNÇÃO } & \multicolumn{3}{|c|}{ PARÂMETROS AJUSTADOS } & \multirow[b]{2}{*}{$\mathbf{R}_{\text {aj }}^{2}$} & \multirow[b]{2}{*}{$\mathrm{S}_{\mathrm{yx}} \%$} & \multirow[b]{2}{*}{$\mathbf{F}$} & \\
\hline & $\mathbf{b}_{0}$ & $b_{1}$ & $b_{2}$ & & & & \\
\hline Prodan & $-0,3528$ & 0,6172 & 0,0129 & 0,8964 & 12,3217 & $37.084,0$ & ** \\
\hline Chapman-Richards & 35,0000 & 0,0589 & 1,1069 & 0,8892 & 12,7436 & $51.955,4$ & ** \\
\hline Schumacher & 35,6240 & $-8,7092$ & - & 0,8528 & 14,6894 & $38.918,2$ & ** \\
\hline
\end{tabular}

Analisando-se os valores das estatísticas de regressão obtidas para os ajustes das funções, verifica-se que as funções de Prodan e de Chapman-Richards, apresentaram diferenças muito pequenas em relação ao coeficiente de determinação (R2aj) e ao erro padrão de estimativa (Syx\%), ficando praticamente empatadas.

A FIGURA 3 apresenta os gráficos de ajuste e de dispersão dos resíduos (em \%) decorrentes dos ajustes das três funções de sítio avaliadas. É possível observar que os gráficos referentes às funções de Prodan e de Chapman-Richards são muito similares, indicando que ambas as funções possuem uma leve tendência a superestimar as alturas, corroborando o que foi evidenciado por meio das estatísticas de regressão.

Já a performance da função de Schumacher, que resultou em estatísticas de regressão inferiores aos das duas demais funções, pode ser considerada menos adequada, não só pela análise dos gráficos de ajuste e de dispersão dos resíduos, como também, pela sua curva resultante, uma vez que possui uma leve tendência a superestimar os valores das alturas dominantes em idades intermediárias e a subestimar as alturas tanto em idades iniciais como em idades próximas à assíntota.

No entanto, sob o ponto de vista da assíntota das curvas quando estimadas para idades um pouco além da abrangência dos dados, a função de Prodan convergiu para valores assintóticos um pouco elevados, enquanto que a função de Schumacher convergiu para valores assintóticos um pouco baixos, sendo ambos considerados inadequados para as condições gerais dos povoamentos de Pinus taeda no Sul do Brasil.

Assim, uma vez que a função de Chapman-Richards apresentou bom ajuste ao conjunto de dados, evidenciado tanto pelas estatísticas de regressão como pela análise dos gráficos de ajuste, de dispersão de resíduos e da curva resultante, esta função foi considerada bastante adequada e satisfatória para a estimativa da altura dominante (em metros) em função da idade (em anos) para o conjunto de dados, cuja equação da curva guia obtida é:

$$
h_{d o m}=35,00 *\left[1-e^{\left(-0,0589464425^{*} I\right)}\right]^{1,1069349251}+\varepsilon_{i}
$$

em que: $h_{\text {dom }}=$ altura dominante $(m) ; I=$ idade (anos) $; e=$ constante de Euler $; \varepsilon_{i}=$ erro

A função de Chapman-Richards também foi utilizada satisfatoriamente para o ajuste de curvas de índice de sítio nos seguintes estudos: Lenhart, Hunt e Blackard (1986) e Zarnoch e Feduccia (1984), para povoamentos de Pinus elliottii e Pinus taeda, no oeste do Estado do Texas nos Estados Unidos; Scolforo e Machado (1988a; 1988b), para plantações de Pinus elliottii e Pinus taeda, respectivamente, 

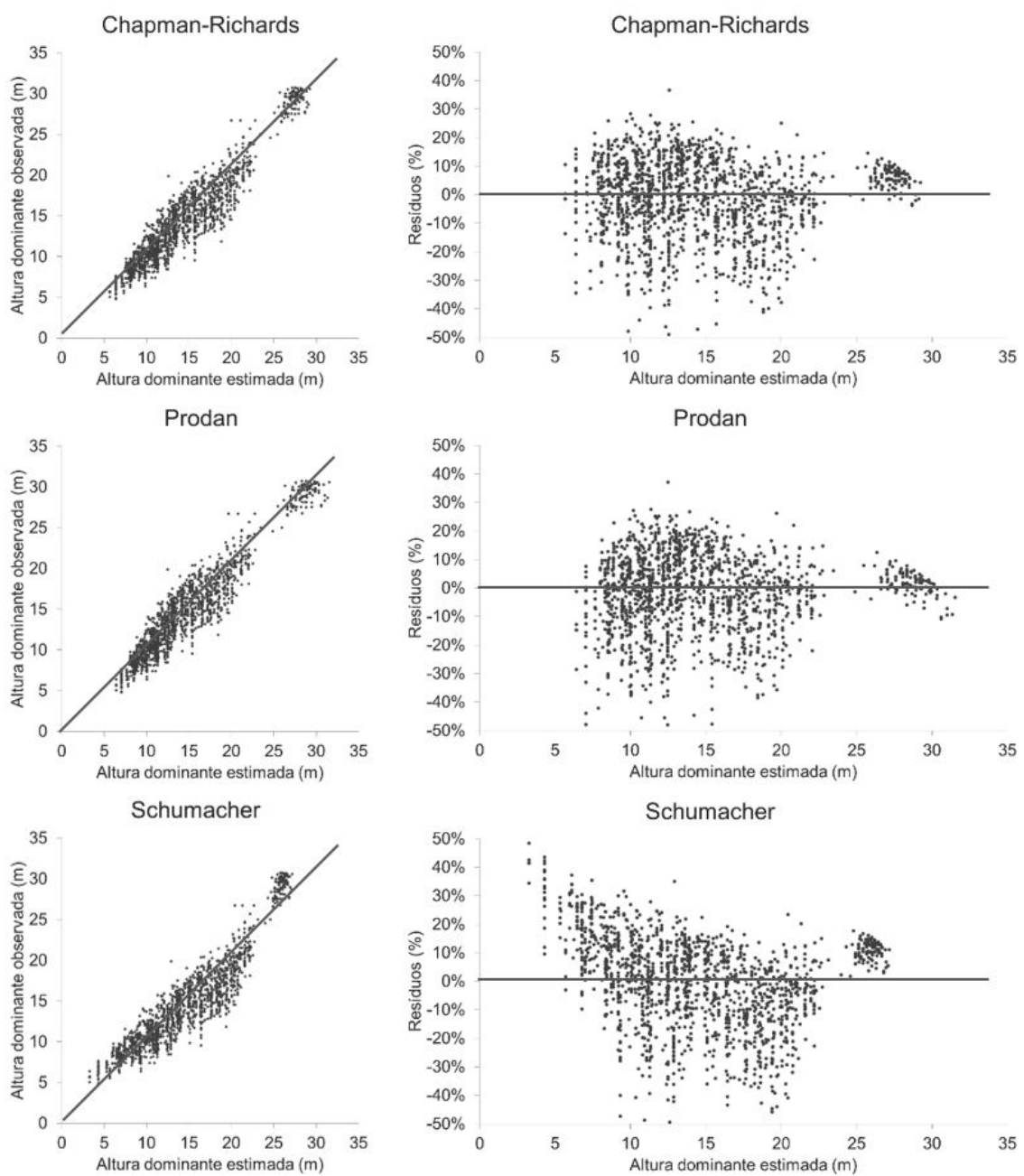

FIGURA 3 -GRÁFICOS DE AJUSTE E DE DISPERSÃO DOS RESÍDUOS (\%) DOS MODELOS DE SÍTIO AJUSTADOS FIGURE 3 - FITTING GRAPH AND RESIDUES SCATTER PLOT (\%) OF FITTED SITE MODELS

para os Estados do Paraná e Santa Catarina; e, Selle (1994) para plantações de Pinus taeda, na região de Cambará do Sul, no Rio Grande do Sul.

Os valores de altura dominante à idade índice de 15 anos variaram de 14,5 a 26,7 metros, com amplitude de 12,2 metros. Contudo, considerou-se o intervalo entre 14,0 e 27,0 metros, tendo sido projetadas 5 classes de sítio, com intervalos de 2,6 metros entre as curvas, detalhadas da seguinte forma:

- classe I: sítio com potencial de crescimento excelente, o melhor entre os existentes em termos de produtividade florestal expressa em função da altura dominante;

- classe II: sítio com bom potencial de crescimento, acima da média de produtividade regional;

- classe III: sítio com potencial de crescimento intermediário, dentro dos padrões médios da região;

- classe IV: sítio com potencial ruim em termos de produtividade expressa pela variável altura dominante, abaixo da média da região;

- classe V: sítio com péssimo potencial, muito aquém da produtividade média da região.

Para a estimativa dos limites inferior e superior para cada idade (TABELA 3), bem como dos limites das curvas em altura para cada classe de sítio (FIGURA 4), a função de Chapman-Richards foi rearranjada, da seguinte forma, assumindo os diferentes índices de sítio $(S)$ :

$$
h_{\text {dom }}=S *\left[\frac{1-e^{\left(-0,0589464425^{*} I\right)}}{1-e^{\left(-0,0589464425^{*} I_{b}\right)}}\right]^{l, 1069349251}+\varepsilon_{i}
$$

em que: $h_{\text {dom }}=$ altura dominante $(m) ; S=$ limites das classes de indice de sitio $(\mathrm{m}) ; I=$ idade $($ anos $) ; I_{b}=$ idade indice $(15$ anos $)$; $e=$ constante de Euler ; $\varepsilon_{i}=$ erro 
Schuchovski et al. - Altura dominante e índice de sítio para Pinus taeda em dois polos de desenvolvimento florestal no sul do Brasil

TABELA 3 - LIMITES DAS CLASSES DE SÍTIO

TABLE 3 - SITE CLASSES LIMITS

\begin{tabular}{|c|c|c|c|c|c|c|c|c|c|c|c|}
\hline \multirow{2}{*}{$\begin{array}{c}\begin{array}{c}\text { IDADE } \\
\text { (anos) }\end{array} \\
1\end{array}$} & \multicolumn{2}{|c|}{ CLASSE V } & \multicolumn{2}{|c|}{ CLASSE IV } & \multicolumn{2}{|c|}{ CLASSE III } & \multicolumn{3}{|c|}{ CLASSE II } & \multicolumn{2}{|c|}{ CLASSE I } \\
\hline & 1,02 & 1,21 & 1,21 & 1,40 & 1,40 & 1,59 & 1,59 & 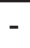 & 1,78 & 1,78 & 1,97 \\
\hline 2 & 2,27 & 2,69 & 2,69 & 3,11 & 3,11 & 3,53 & 3,53 & - & 3,95 & 3,95 & 4,37 \\
\hline 3 & 3,53 & 4,19 & 4,19 & 4,84 & 4,84 & 5,50 & 5,50 & - & 6,15 & 6,15 & 6,81 \\
\hline 4 & 4,76 & 5,65 & 5,65 & 6,53 & 6,53 & 7,42 & 7,42 & - & 8,30 & 8,30 & 9,18 \\
\hline 5 & 5,94 & 7,04 & 7,04 & 8,14 & 8,14 & 9,25 & 9,25 & - & 10,35 & 10,35 & - 11,45 \\
\hline 6 & 7,05 & 8,35 & 8,35 & 9,66 & 9,66 & 10,97 & 10,97 & - & 12,28 & 12,28 & - 13,59 \\
\hline 7 & 8,08 & 9,58 & 9,58 & 11,08 & 11,08 & 12,58 & 12,58 & - & 14,09 & 14,09 & - $\quad 15,59$ \\
\hline 8 & 9,05 & - $\quad 10,73$ & 10,73 & 12,41 & 12,41 & 14,09 & 14,09 & - & 15,77 & 15,77 & - 17,45 \\
\hline 9 & 9,94 & - $\quad 11,79$ & 11,79 & 13,63 & 13,63 & 15,48 & 15,48 & - & 17,32 & 17,32 & - $\quad 19,17$ \\
\hline 10 & 10,77 & $-\quad 12,77$ & 12,77 & 14,77 & 14,77 & - $\quad 16,76$ & 16,76 & - & 18,76 & 18,76 & - 20,76 \\
\hline 11 & 11,53 & - $\quad 13,67$ & 13,67 & 15,81 & 15,81 & - $\quad 17,95$ & 17,95 & - & 20,09 & 20,09 & $\begin{array}{l}-\quad 22,23 \\
\end{array}$ \\
\hline 12 & 12,23 & $-\quad 14,50$ & 14,50 & 16,77 & 16,77 & 19,04 & 19,04 & - & 21,31 & 21,31 & - $\quad 23,58$ \\
\hline 13 & 12,87 & $-\quad 15,26$ & 15,26 & 17,65 & 17,65 & - 20,04 & 20,04 & - & 22,43 & 22,43 & - 24,82 \\
\hline 14 & 13,46 & - $\quad 15,96$ & 15,96 & 18,46 & 18,46 & $-\quad 20,96$ & 20,96 & - & 23,46 & 23,46 & - 25,96 \\
\hline 15 & 14,00 & $-\quad 16,60$ & 16,60 & 19,20 & 19,20 & 21,80 & 21,80 & - & 24,40 & 24,40 & - $\quad 27,00$ \\
\hline 16 & 14,49 & - $\quad 17,19$ & 17,19 & 19,88 & 19,88 & 22,57 & 22,57 & - & 25,26 & 25,26 & - 27,95 \\
\hline 17 & 14,95 & - $\quad 17,72$ & 17,72 & 20,50 & 20,50 & 23,27 & 23,27 & - & 26,05 & 26,05 & - $\quad 28,83$ \\
\hline 18 & 15,36 & - $\quad 18,21$ & 18,21 & 21,06 & 21,06 & - $\quad 23,92$ & 23,92 & - & 26,77 & 26,77 & - 29,62 \\
\hline 19 & 15,74 & $-\quad 18,66$ & 18,66 & 21,58 & 21,58 & 24,50 & 24,50 & - & 27,43 & 27,43 & - $\quad 30,35$ \\
\hline 20 & 16,08 & - $\quad 19,07$ & 19,07 & 22,05 & 22,05 & $-25,04$ & 25,04 & - & 28,03 & 28,03 & - $\quad 31,01$ \\
\hline 21 & 16,40 & - $\quad 19,44$ & 19,44 & 22,48 & 22,48 & $-\quad 25,53$ & 25,53 & 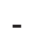 & 28,57 & 28,57 & - $\quad 31,62$ \\
\hline 22 & 16,68 & - $\quad 19,78$ & 19,78 & 22,88 & 22,88 & $-\quad 25,98$ & 25,98 & - & 29,07 & 29,07 & $\begin{array}{l}-\quad 32,17\end{array}$ \\
\hline 23 & 16,94 & - 20,09 & 20,09 & 23,24 & 23,24 & - 26,38 & 26,38 & - & 29,53 & 29,53 & - $\quad 32,68$ \\
\hline 24 & 17,18 & - $\quad 20,37$ & 20,37 & 23,56 & 23,56 & 26,75 & 26,75 & - & 29,94 & 29,94 & - $\quad 33,13$ \\
\hline 25 & 17,40 & - $\quad 20,63$ & 20,63 & 23,86 & 23,86 & - $\quad 27,09$ & 27,09 & - & 30,32 & 30,32 & - $\quad 33,55$ \\
\hline 26 & 17,60 & - $\quad 20,86$ & 20,86 & 24,13 & 24,13 & - 27,40 & 27,40 & 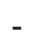 & 30,67 & 30,67 & $\begin{array}{l}-\quad 33,93 \\
\end{array}$ \\
\hline 27 & 17,78 & - $\quad 21,08$ & 21,08 & 24,38 & 24,38 & - $\quad 27,68$ & 27,68 & - & 30,98 & 30,98 & - $\quad 34,28$ \\
\hline 28 & 17,94 & $-\quad 21,27$ & 21,27 & 24,60 & 24,60 & - $\quad 27,93$ & 27,93 & - & 31,26 & 31,26 & - $\quad 34,60$ \\
\hline 29 & 18,09 & - $\quad 21,45$ & 21,45 & 24,81 & 24,81 & $-\quad 28,17$ & 28,17 & - & 31,52 & 31,52 & - $\quad 34,88$ \\
\hline 30 & 18,22 & - $\quad 21,61$ & 21,61 & 24,99 & 24,99 & - $\quad 28,38$ & 28,38 & - & 31,76 & 31,76 & - $\quad 35,15$ \\
\hline 31 & 18,35 & $-\quad 21,75$ & 21,75 & 25,16 & 25,16 & $-\quad 28,57$ & 28,57 & - & 31,98 & 31,98 & $\begin{array}{l}-\quad 35,38\end{array}$ \\
\hline 32 & 18,46 & - $\quad 21,89$ & 21,89 & 25,32 & 25,32 & $-\quad 28,74$ & 28,74 & 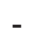 & 32,17 & 32,17 & - $\quad 35,60$ \\
\hline 33 & 18,56 & - 22,01 & 22,01 & 25,46 & 25,46 & - $\quad 28,90$ & 28,90 & & 32,35 & 32,35 & - $\quad 35,80$ \\
\hline 34 & 18,65 & - 22,12 & 22,12 & 25,58 & 25,58 & $-\quad 29,05$ & 29,05 & - & 32,51 & 32,51 & - $\quad 35,98$ \\
\hline 35 & 18,74 & $\begin{array}{l}-\quad 22,22 \\
\end{array}$ & 22,22 & 25,70 & 25,70 & - 29,18 & 29,18 & - & 32,66 & 32,66 & - $\quad 36,14$ \\
\hline
\end{tabular}

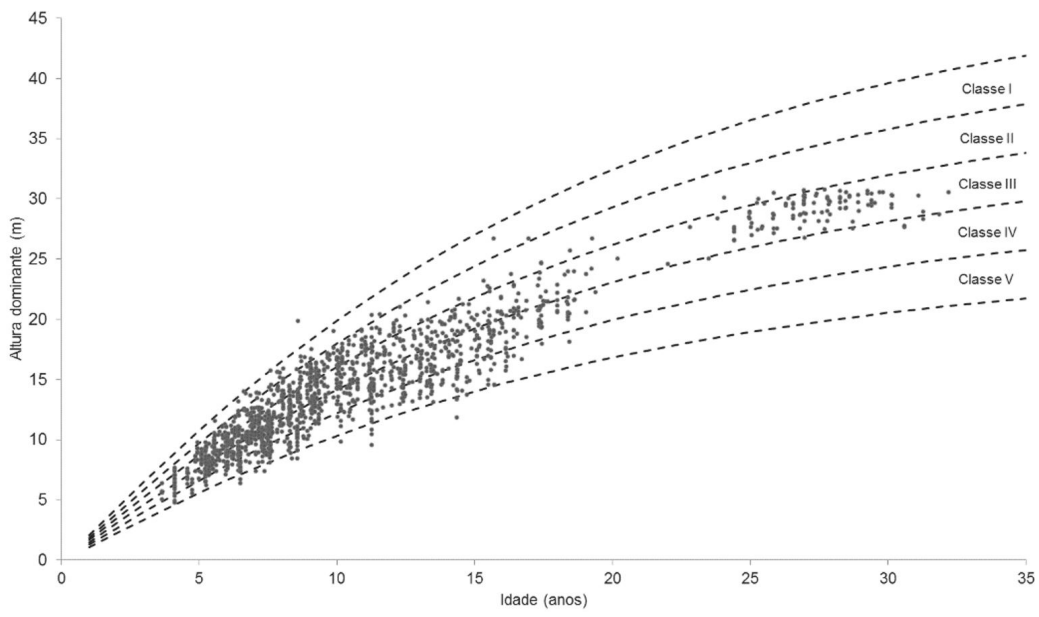

FIGURA 4 - CURVAS DE LIMITE DAS CLASSES DE SÍTIO ESTIMADAS COM O MODELO DE CHAPMAN-RICHARDS (RICHARDS, 1959; CHAPMAN, 1961)

FIGURE 4 - SITE INDEX LIMIT CURVES ESTIMATED WITH CHAPMAN-RICHARDS (RICHARDS, 1959; CHAPMAN, 1961) MODEL 
E, as parcelas do estudo foram classificadas quanto ao sítio, em função de sua altura dominante, com a seguinte função:

$$
S=h_{d o m} *\left[\frac{1-e^{\left(-0,0589464425^{*} I_{b}\right)}}{1-e^{\left(-0,0589464425^{*} I\right)}}\right]^{l, 1069349251}+\varepsilon_{i}
$$

em que: $h_{\text {dom }}=$ altura dominante $(m) ; S=$ indice de sitio $(m) ; I=$ idade $($ anos $) ; I_{b}=$ idade indice (anos); e=constante de Euler; $\varepsilon_{i}=$ erro

A confirmação do anamorfismo destas curvas seguiu a metodologia utilizada por Kirby (1975), Scolforo (1992), Scolforo e Machado (1988a, 1988b), sendo constatada mediante a verificação das duas seguintes premissas:

I. similaridade entre os coeficientes de variação (em porcentagem) das alturas médias das árvores dominantes por classe de sítio e idade; e

II. existência de relação linear entre os índices de sítio e as alturas dominantes médias nas várias idades consideradas, expressando que o índice de sítio não depende da idade, mas, sim, da capacidade produtiva do local.

Analisando-se os valores dos coeficientes de variação (\%) das alturas médias das árvores dominantes, apresentados na TABELA 4, observa-se que estes não ultrapassam 10\%, mantendo-se relativamente constante ao longo das idades, para as 5 classes de sítio, não havendo evidências de disparidade nos valores, atendendo à primeira das premissas para confirmar o anamorfismo das curvas.

TABELA 4 - COEFICIENTES DE VARIAÇÃO (\%) DAS ALTURAS MÉDIAS DAS ÁRVORES DOMINANTES POR CLASSE DE SÍTIO E IDADE

TABLE 4 - DOMINANT TREE AVERAGE HEIGHT COEFFICIENT OF VARIATION (\%) PER SITE CLASS AND AGE

\begin{tabular}{|c|c|c|c|c|c|c|c|c|c|c|c|c|c|c|c|}
\hline \multirow{2}{*}{$\begin{array}{c}\text { CLASSE } \\
\text { SÍTIO }\end{array}$} & \multicolumn{15}{|c|}{ COEFICIENTE DE VARIAÇÃO (\%) POR IDADE (anos) } \\
\hline & 3 & 4 & 5 & 6 & 7 & 8 & 9 & 10 & 11 & 12 & 13 & 14 & 15 & 16 & 17 \\
\hline 1 & - & - & - & 4,92 & 5,86 & 9,24 & 6,60 & - & 0,23 & - & - & - & - & - & - \\
\hline II & - & 9,08 & 6,49 & 5,75 & 5,98 & 5,26 & 4,14 & 4,90 & 3,36 & 3,24 & 5,19 & - & 1,37 & - & 0,24 \\
\hline III & 7,63 & 8,60 & 6,14 & 5,26 & 4,49 & 4,45 & 4,55 & 4,96 & 3,79 & 3,13 & 3,70 & 3,75 & 4,12 & 4,10 & 4,17 \\
\hline IV & 6,42 & 7,44 & 5,18 & 5,64 & 4,75 & 4,29 & 4,08 & 5,61 & 4,32 & 4,21 & 4,82 & 4,47 & 4,43 & 5,10 & 3,49 \\
\hline $\mathrm{V}$ & - & 8,18 & 6,28 & 6,57 & 6,63 & 6,70 & 1,89 & 6,90 & 9,39 & 4,17 & 4,46 & 7,06 & 5,54 & 3,10 & - \\
\hline \multirow{2}{*}{$\begin{array}{l}\text { CLASSE } \\
\text { SÍTIO }\end{array}$} & \multicolumn{15}{|c|}{ COEFICIENTE DE VARIAÇÃO (\%) POR IDADE (anos) - continuação } \\
\hline & 18 & 19 & 20 & 21 & 22 & 23 & 24 & 25 & 26 & 27 & 28 & 29 & 30 & 31 & 32 \\
\hline 1 & - & - & - & - & - & - & - & - & - & - & - & - & - & - & 0,00 \\
\hline II & - & - & - & - & - & - & 1,42 & - & - & - & - & - & - & - & 2,45 \\
\hline III & 3,60 & 1,38 & - & 8,36 & & 3,24 & 2,64 & 2,65 & 3,14 & 2,32 & 1,64 & 1,76 & 3,23 & - & - \\
\hline IV & 2,93 & 5,53 & - & - & - & - & - & - & - & - & - & 0,34 & 0,85 & - & - \\
\hline V & - & - & - & - & - & - & - & - & - & - & - & - & - & - & - \\
\hline
\end{tabular}

A FIGURA 5 demonstra a linearidade para as diferentes idades, sendo que os coeficientes de correlação de Pearson entre as variáveis foram superiores a 0,95 para todas as idades, com exceção das idades 4 e 5 anos, conforme evidenciado na TABELA 5, atendendo-se assim a segunda premissa utilizada para a confirmação do anamorfismo das curvas.

O mesmo comportamento anamórfico foi observado por Scolforo e Machado (1988a, 1988b) em plantios de Pinus elliottii var. elliottii e Pinus taeda nos Estados do Paraná e Santa Catarina e por Scolforo (1992) em povoamentos de Pinus caribaea var. hondurensis no Estado de São Paulo.

Com a finalidade de comprovar a eficiência das curvas construídas, verificou-se a estabilidade das alturas dominantes das parcelas ao longo das curvas que expressam as classes de sítio, graficamente apresentada na FIGURA 6, conforme metodologia adotada em Machado (1980), Machado et al. (1997), Scolforo (1992), Scolforo e Machado (1988a, 1988b). Para melhor visualização, foram utilizados dados de parte das parcelas envolvidas no trabalho.

Parcelas que se encontram próximas aos limites da classe de sítio podem flutuar de um sítio para outro na medida em que haja qualquer mudança climática ou de qualquer outro fator do meio que influencie seu desenvolvimento. No entanto, a estabilidade do valor do índice de sítio atribuído a uma parcela durante o período de observação foi constatada na grande maioria das situações testadas; 
Schuchovski et al. - Altura dominante e índice de sítio para Pinus taeda em dois polos de desenvolvimento florestal no sul do Brasil

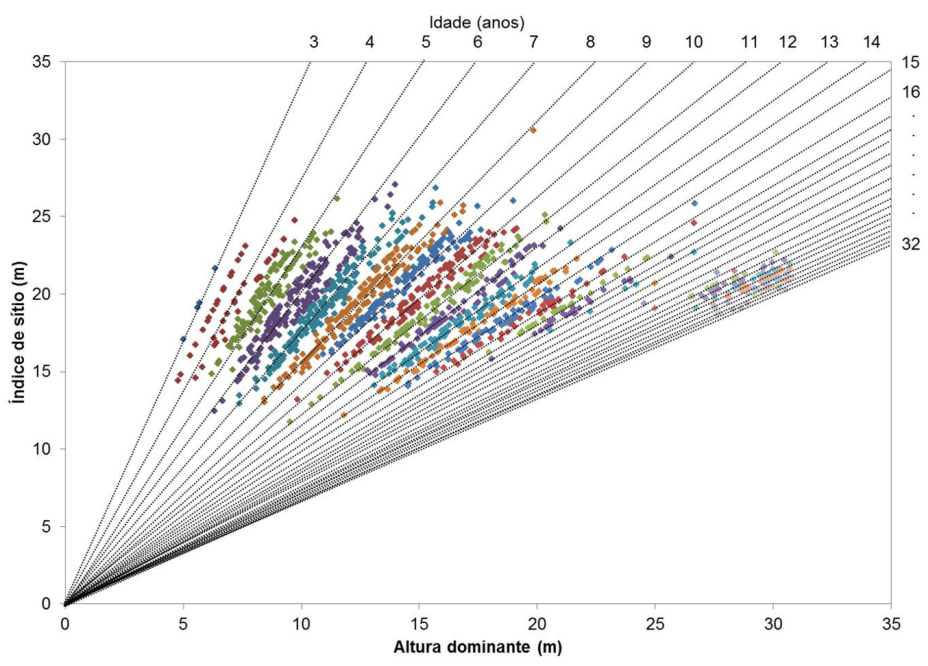

FIGURA 5 - CORRELAÇÃO ENTRE ÍNDICE DE SÍTIO E ALTURAS DOMINANTES MÉDIAS PARA AS DIFERENTES IDADES FIGURE 5 - CORRELATION BETWEEN SITE INDEX AND AVERAGE DOMINANT HEIGHTS FOR DIFFERENT AGES

TABELA 5 - CORRELAÇÃO ENTRE ÍNDICE DE SÍTIO E ALTURA DOMINANTE (COEFICIENTE DE CORRELAÇÃO DE PEARSON) POR IDADE

TABLE 5 - CORRELATION BETWEEN SITE INDEX AND DOMINANT HEIGHT (PEARSON CORRELATION COEFFICIENT) PER AGE

\begin{tabular}{ccccccccccccccc}
\hline \multicolumn{110}{c}{ IDADE (anos) } \\
\hline $\mathbf{3}$ & $\mathbf{4}$ & $\mathbf{5}$ & $\mathbf{6}$ & $\mathbf{7}$ & $\mathbf{8}$ & $\mathbf{9}$ & $\mathbf{1 0}$ & $\mathbf{1 1}$ & $\mathbf{1 2}$ & $\mathbf{1 3}$ & $\mathbf{1 4}$ & $\mathbf{1 5}$ & $\mathbf{1 6}$ & $\mathbf{1 7}$ \\
1,000 & 0,892 & 0,943 & 0,956 & 0,979 & 0,983 & 0,983 & 0,983 & 0,992 & 0,993 & 0,991 & 0,996 & 0,995 & 0,997 & 0,999 \\
\hline \multicolumn{10}{c}{} & \multicolumn{10}{c}{ IDADE (anos) - continuação } \\
\hline $\mathbf{1 8}$ & $\mathbf{1 9}$ & $\mathbf{2 0}$ & $\mathbf{2 1}$ & $\mathbf{2 2}$ & $\mathbf{2 3}$ & $\mathbf{2 4}$ & $\mathbf{2 5}$ & $\mathbf{2 6}$ & $\mathbf{2 7}$ & $\mathbf{2 8}$ & $\mathbf{2 9}$ & $\mathbf{3 0}$ & $\mathbf{3 1}$ & $\mathbf{3 2}$ \\
0,995 & 0,999 & - & - & 1,000 & 1,000 & 0,985 & 0,987 & 0,992 & 0,991 & 0,993 & 0,987 & 1,000 & 0,995 & - \\
\hline
\end{tabular}

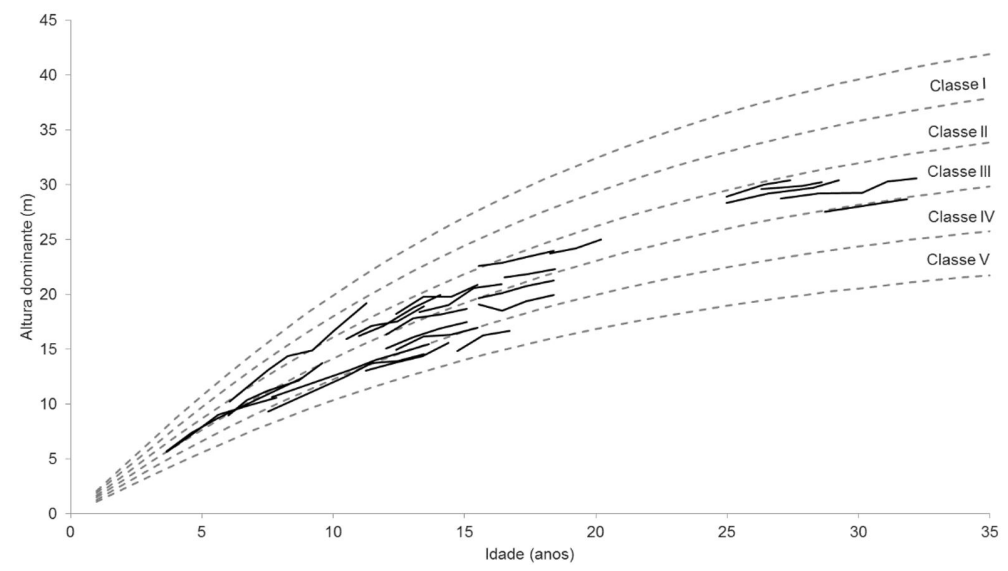

FIGURA 6 - EFICIÊNCIA DAS CURVAS DE ÍNDICE DE SÍTIO AJUSTADAS FIGURE 6 - FITTED SITE CURVES EFFICIENCY

e, embora algumas parcelas analisadas tenham passado de uma classe para a outra ao longo das remedições, de modo geral, os resultados obtidos nesta análise foram satisfatórios e confirmam o exposto por Clutter et al. (1984), que a essência na confecção das curvas de sítio é a altura média das árvores dominantes da parcela permanecer na mesma classe de sítio durante toda sua vida, de modo que esta classificação propicie uma base de consistência e confiabilidade às curvas de índice de sítio e aos modelos de crescimento e produção.

A FIGURA 7 apresenta a comparação da curva de sítio para Pinus taeda obtida neste estudo com a função de Chapman-Richards e as curvas obtidas em estudos anteriores para a mesma espécie:

a) Machado (1980), com a função de Prodan, para a região central do Estado do Paraná, município de Telêmaco Borba, com idade índice de 10 anos; 


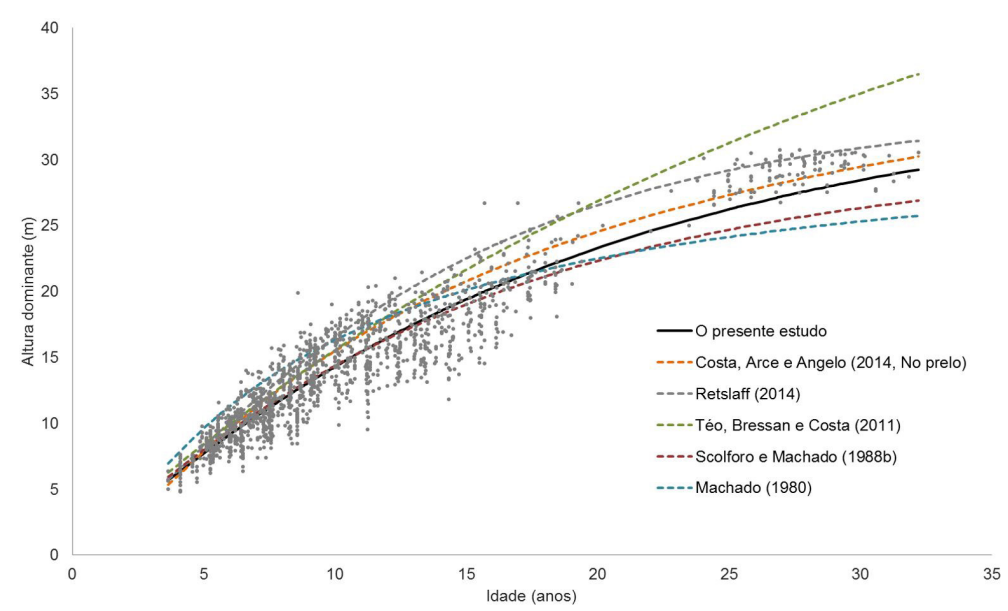

FIGURA 7 - COMPARAÇÃO ENTRE DIFERENTES CURVAS DE ÍNDICE DE SÍTIO PARA Pinus taeda PARA A REGIÃO SUL DO BRASIL

FIGURE 7 - COMPARISON BETWEEN DIFFERENT SITE INDEX CURVES FOR LOBLOLLY PINE (Pinus taeda) FOR THE SOURTHERN REGION OF BRASIL

$$
h_{\text {dom }}=\frac{I^{2}}{0,60323+0,2464 * I+0.03061 * I^{2}}
$$

b) Scolforo e Machado (1988a), que utilizaram a função de Chapman-Richards para os Estados do Paraná e Santa Catarina, com idade índice de 25 anos;

$$
h_{\text {dom }}=30,1750 *\left[1-e^{(-0,072009 * I)}\right]^{1,11150}
$$

c) Téo, Bressan e Costa (2011), que utilizaram a função monomolecular, para a região de Caçador, Estado de Santa Catarina;

$$
h_{\text {dom }}=56,59182 *\left[1-e^{\left(-0,032165^{*} I\right)}\right]
$$

d) Retslaff (2014), que utilizou a função de Chapman-Richards para o Estado do Paraná e Santa Catarina, com idade índice de 18 anos;

$$
h_{d o m}=33,843900 *\left[1-e^{\left(-0,0925913^{*}\right)}\right]^{1,419870}
$$

e) Costa, Arce e Angelo (2014), com a função de Prodan para a região de Volta Grande, Rio Negrinho, Santa Catarina, com idade índice de 18 anos.

$$
h_{\text {dom }}=\frac{I^{2}}{1,0225+0,323 * I+0,02204 * I^{2}}
$$

em que: $h_{\text {dom }}=$ altura dominante $(m) ; I=$ idade (anos) $; e=$ constante de Euler

Pela análise e comparação das curvas de índice de sítio ajustadas para a mesma espécie, observa-se que, apesar do formato sigmoidal, de modo geral, o padrão das curvas difere bastante. Entre as curvas, a que mais se assemelha à ajustada neste estudo, é a de Costa, Arce e Angelo (2014), apesar da pequena superioridade desta ao longo de todo o traçado, em relação àquela.

A curva de Scolforo e Machado (1988a) possui valores iniciais de alturas dominantes muito semelhantes aos observados neste estudo, mas os valores assintóticos são menores. Por outro lado, a curva de Machado (1980) estima maiores valores de alturas para as idades iniciais do que a resultante deste estudo e menores alturas para as fases mais avançadas da floresta, resultando também em valor assintótico menor do que o deste estudo. Estas evidências podem ser explicadas pela evolução dos plantios ao longo destes anos, tanto em relação aos tratos silviculturais como à qualidade do material genético utilizado.

A curva de Retslaff (2014) possui comportamento similar à ajustada no presente estudo, mas indica alturas dominantes maiores para todas as idades, sobretudo nas idades intermediárias. 
Schuchovski et al. - Altura dominante e índice de sítio para Pinus taeda em dois polos de desenvolvimento florestal no sul do Brasil

Já a curva de Téo, Bressan e Costa (2011) estima valores de altura superiores a quase todas as outras curvas, sobretudo para as idades mais avançadas, atingindo um valor assintótico bastante superior. Este fato pode ser explicado pela superioridade de qualidade de solos observada na região do estudo supracitado e, portanto, sítio.

Como se pode observar, as curvas utilizadas na comparação abrangem pequenas porções de povoamentos florestais de Pinus taeda na Região Sul do Brasil. Seria muito interessante e valioso para todo o setor de florestas plantadas se a curva de índice de sítio para plantações de Pinus taeda pudesse ser consolidada, para toda a Região Sul do Brasil, compreendendo dados de inventário florestal de mais povoamentos na Região, com maior abrangência e variabilidade de dados tanto em termos de idade como de produtividade.

\section{CONCLUSÃO}

O modelo de Chapman-Richards foi o que melhor caracterizou os povoamentos de Pinus taeda avaliados no estudo, considerado superior aos modelos de Prodan e de Schumacher, sendo utilizado para classificar o sítio das parcelas.

As curvas de índice de sítio para Pinus taeda para os polos de desenvolvimento florestal de Jaguariaíva e de Rio Negrinho apresentam um padrão anamórfico.

Ao realizar comparações entre as curvas de sítio geradas em estudos anteriores, para a mesma espécie e região similares, é possível concluir que as plantações de Pinus taeda sofreram alterações no padrão de crescimento, uma vez que a curva obtida neste estudo é superior às curvas de estudos mais antigos, tais como Machado (1980) e Scolforo e Machado (1988a).

\section{AGRADECIMENTOS}

À empresa Valor Florestal (Global Forest Partners - GFP), pelo fornecimento da base de dados que propiciaram o desenvolvimento deste estudo.

\section{REFERÊNCIAS BIBLIOGRÁFICAS}

AHRENS, S. A seleção simultânea do ótimo regime de desbastes e da idade de rotação, para povoamentos de Pinus taeda L., através de um modelo de programação dinâmica. 1992. 189 p. Tese (Doutorado em Ciências Florestais) - Setor de Ciências Agrárias, Universidade Federal do Paraná, Curitiba, 1992.

ARCE, J. E. Um sistema de análise, simulação e otimização do sortimento florestal em função da demanda por multiprodutos e dos custos de transporte. 2000. 125 p. Tese (Doutorado em Ciências Florestais) Setor de Ciências Agrárias, Universidade Federal do Paraná, Curitiba, 2000.

ARCE, J. E.; GOMES, F. S.; SANQUETTA, C. R.; CRUZ, E. C. Utilização dos processos de difusão na avaliação e simulação precoces do crescimento de povoamentos de Pinus taeda L. Cerne, Lavras, v. 4, n. 1, p. 157-170, 1998.

ARCE, J. E.; PIZZATO, W.; SANQUETTA, C. R.; WENDLING, J. L. G.; MAESTRI, R. Utilização das matrizes de transição na avaliação e simulação precoces do crescimento de povoamentos de Pinus taeda L. Revista Floresta, Curitiba, v. 27, n. 1/2, p. 83-98, 1997.

ASSMANN, E. The principles of forest yield study: studies in the organic production, structure, increment and yield of forest stands. Oxford: Pergamon, 1970.

AVERY, T. E.; BURKHART, H. E. Forest measurements. 5. ed. New York: McGraw-Hill, 2002.

BOGNOLA, I. A. Unidades de manejo para Pinus taeda L. no planalto norte catarinense, com base em características do meio físico. 160 f. Tese (Doutorado em Ciências Florestais) - Setor de Ciências Agrárias, Universidade Federal do Paraná, Curitiba, 2007.

BRASIL. Ministério do Meio Ambiente -MMA. Ministério do Planejamento, Orçamento e Gestão. Mapa de biomas do Brasil: primeira aproximação. Brasília: IBGE, 2004a. Escala 1:5.000.000. Disponível em: <ftp:// ftp.ibge.gov.br/Cartas_e_Mapas/Mapas_Murais/ >. Acesso em: 16 ago 2017. 
BRASIL. Ministério do Planejamento, Orçamento e Gestão. Mapa de vegetação do Brasil. Brasília: IBGE, 2004b. Escala 1:5.000.000. Disponível em: < ftp://ftp.ibge.gov.br/Cartas_e_Mapas/Mapas_Murais/ >. Acesso em: 16/ ago 2017.

BURKHART, H. E.; TOMÉ, M. Modeling forest trees and stands. Dordrecht: Springer, 2012.

CHAPMAN, D. G. Statistical problems in dynamics of exploited fisheries population. In: FOURTH BERKELEY SYMPOSIUM ON MATHEMATICAL STATISTICS AND PROBABILITY, 4.,1961, Berkeley. Proceedings... Berkeley: University of California Press, 1961. p. 153-168.

CLUTTER, J. L.; HARMS, W. R.; BRISTER, G. H.; RHENEY, J. W. Stand structure and yields of site-prepared loblolly pine plantations in the lower coastal plain of the Carolinas, Georgia, and North Florida. Ashville: USDA, Forest Service, Southeastern Forest Experimental Station, 1984. 173 p. (General Technical Report SE-27).

CLUTTER, J. L.; FORTSON, J. C.; PIENAAR, L. V.; BRISTER, G. H.; BAILEY, R. L. Timber management: a quantitative approach. New York: John Wiley, 1983.

COSTA, E. R. O.; ARCE, J. E.; ANGELO, A. C. Curvas de índice de sítio para Pinus taeda L. para a região de Volta Grande, no município de Rio Negrinho, Santa Catarina. No prelo. 2014.

CUNHA NETO, F. R.; SCOLFORO, J. R. S.; CALEGARIO, N.; OLIVEIRA, A. D. de; KANEGAE JÚNIOR, H. Modelo para predição da produção por classe de diâmetro para Eucalyptus grandis. Cerne, Lavras, v. 1, n. 1, p. 108-122, 1994.

EMBRAPA - EMPRESA BRASILEIRA DE PESQUISA AGROPECUÁRIA. Centro Nacional de Pesquisa de Solos. Mapa de solos do estado do Paraná: 2008. Escala 1:600.000. Disponível em: < http://www.cnps.embrapa. br/noticias/banco_noticias/110707.html >. Acesso em: 23 set. 2013.

KÖPPEN, W. Das geographisca system der klimate. In: KOPPEN, W.; GEIGER, G. Handbuch der klimatologie. Berlin: Gebruder Borntraeger, 1936. p. 44 p.

IBÁ - INDÚSTRIA BRASILEIRA DE ÁRVORES. Relatório 2016. IBÁ: Brasília, 2016.

IAPAR - INSTITUTO AGRONÔMICO DO PARANÁ. Cartas climáticas do Estado do Paraná. Londrina: IAPAR, 2000. Disponível em: < http://www.iapar.br/modules/conteudo/conteudo.php?conteudo=677 >. Acesso em: 16 ago 2017.

KIRBY, C. L. Site index equations for lodgepole pine and white spruce in Alberta. Edmonton: Canadian Forestry Service / Northern Forest Research Centre, 1975. 12 p. (Information Report NOR-X-142).

LENHART, J. D.; HUNT, E. V.; BLACKARD, J. A .Site index equations for loblolly and slash pine plantations on non-old-fields in East Texas, Southern Journal of Applied Forestry, Washington, v. 10, n. 2, p. 109-112, 1986.

MACHADO, S. A. Curvas de índice de sítio para plantações de Pinus taeda L. na região central do estado do Paraná. Floresta, Curitiba, v. 11. n. 2, p. 4-18, 1980.

MACHADO, S. A.; OLIVEIRA, E. B.; CARPANEZZI, A. P.; BARTOSZECK, A. C. P. S. Classificação de sítio para bracatingais na região metropolitana de Curitiba. Boletim de Pesquisa Florestal, Colombo, n. 35, p. 21-37, 1997.

OLIVEIRA, E. B. de. Um sistema computadorizado de prognose do crescimento e produção de Pinus taeda L., com critérios quantitativos para a avaliação técnica e econômica de regimes de manejo. 134 p. Tese (Doutorado em Ciências Florestais) - Setor de Ciências Agrárias, Universidade Federal do Paraná, Curitiba, 1995.

OLIVEIRA, Y. M. M.; AHRENS, S. Curvas de índice de sítio para povoamentos de Pinus elliottii var. elliottii Engelm. e P. taeda L. estabelecidos no sul e sudeste do Brasil: resultados preliminares. Curitiba: EMBRAPA-CNPF, 1987. 11 p. (Circular Técnica, 14).

OLIVEIRA, Y. M. M.; OLIVEIRA, E. B.; HAFLEY, W. L. SisPinus: simulador de crescimento e de produção para plantios de Pinus elliottii e Pinus taeda sob manejo no sul do Brasil. In: ENCONTRO BRASILEIRO DE PLANEJAMENTO FLORESTAL, 1., 1989, Curitiba. Anais... Curitiba: EMBRAPA-CNPF, 1989. p. 107-118.

OLIVER, C. D.; LARSON, B. C.; Forest stand dynamics: update edition. [S.l.]: John Wiley \& Sons, 1996.

Sci. For., Piracicaba, v. 47, n. 121, p. 92-104, mar. 2019 DOI: dx.doi.org/10.18671/scifor.v47n121.09 
Schuchovski et al. - Altura dominante e índice de sítio para Pinus taeda em dois polos de desenvolvimento florestal no sul do Brasil

PRODAN, M. Forest biometrics. Oxford: Pergamon Press, 1968.

RETSLAFF, F. A. S. Simulador para prognose da produção de Pinus taeda com diagrama de manejo da densidade associado à modelagem em classes de diâmetro. 2014. 166 p. Tese (Doutorado em Ciências Florestais) - Setor de Ciências Agrárias, Universidade Federal do Paraná, Curitiba, 2014.

RICHARDS, F. J. A flexible growth function for empirical use. Journal of Experimental Botany, Lancaster, v. 10, n. 2, p. 290-301, 1959.

SANQUETTA, C. R.; BEHLING, A; PELISSARI, A. L.; CORTE, A. P. D.; PÉlliCO NETTO, S.; SIMON, A. A. Probabilistic distributions for Acacia mearnsii De Wild total height and the influence of environmental factors. Journal of Applied Mathematics and Physics, v. 2, p. 1-10, 2014.

SCHUCHOVSKI, M. S. Modelos alométricos e de produção para plantações de Pinus taeda nos estados do Paraná e Santa Catarina. 2014. 182 p. Tese (Doutorado em Ciências Florestais) - Setor de Ciências Agrárias, Universidade Federal do Paraná, Curitiba, 2014.

SCHUCHOVSKI, M. S. Diagnóstico e planejamento do consumo de madeira e da produção em plantações florestais no estado do Paraná. 2003. 78 p. Dissertação (Mestrado em Ciências Florestais) - Setor de Ciências Agrárias, Universidade Federal do Paraná, Curitiba, 2003.

SCHUMACHER, F. X. A new growth curve and its application to timber-yield studies. Journal of Forestry, v. 37, p. 819-820, 1939.

SCOLFORO, J. R. S. Curvas de índice de sítio para Pinus caribaea var. hondurensis. IPEF, n. 45, p. 40-47, 1992.

SCOLFORO, J. R. S.; MACHADO, S. A. Um sistema de crescimento e produção com simulador de desbaste. Scientia Forestalis, Piracicaba, n. 50, p. 51-64, 1996.

SCOLFORO, J. R. S.; MACHADO, S. A. Curvas de índice de sítio para plantações de Pinus taeda nos estados do Paraná e Santa Catarina. Floresta, Curitiba, v. 18, n. 1/2, p. 159-173. 1988 a.

SCOLFORO, J. R. S.; MACHADO, S. do A. Curvas de índice de sítio para plantações de Pinus elliottii nos estados do Paraná e Santa Catarina. Floresta, Curitiba, v. 18, n. 1/2, p. 140-158, 1988b.

SCOLFORO, J. R. S.; MAESTRI, R.; MACHADO, S. A. Um modelo de crescimento e produção como suporte para manejo florestal. In: CURSO DE MANEJO FLORESTAL SUSTENTAVEL, 1., 1997, Curitiba. Tópicos em manejo florestal sustentável. Colombo: EMBRAPA-CNPF, 1997. p. 177-206.

SELLE, G. L.; SCHNEIDER, P. R.; FINGER, C. A. G. Classificação de sítio para Pinus taeda L. através da altura dominante, para a região de Cambará do Sul, RS, Brasil. Ciência Florestal, Santa Maria, v. 4, n. 1, p. 77-95, 1994.

SPURR, S. H. Forest Inventory. New York: Ronald Press, 1952.

STRAND, L. Numerical Constructions of Site-Index Curves. Forest Science, Bethesda, v. 10, n. 4, p. 410-414, 1964.

TEMPS, M. Adição da precipitação pluviométrica na modelagem do crescimento e da produção florestal em povoamentos não desbastados de Pinus taeda L.. 2005. 83 p. Dissertação (Mestrado em Ciências Florestais) - Setor de Ciências Agrárias, Universidade Federal do Paraná, Curitiba, 2005.

TÉO, S. J.; BRESSAN, D. R.; COSTA, R. H. Uso de modelos estatísticos para classificação de sítios em povoamentos de Pinus taeda na região de Caçador - SC. Floresta, Curitiba, v. 41, n. 1, p. 179-188, 2011.

ZARNOCH, S, J.; FEDUCCIA, D. P. Slash pine plantation site index curves for the West Gulf. Southern Journal of Applied Forestry, Washington, v. 8, n. 4, p. 223-225, 1984.

Recebido em: 30/08/2017

Aceito em: 24/07/2018 